\title{
Un état-civil chargé d'enjeux : Saint-George,
}

\section{5-1799}

\section{Luc Nemeth}

\section{(2) OpenEdition \\ 1 Journals}

\section{Édition électronique}

URL : https://journals.openedition.org/ahrf/2130

DOI : 10.4000/ahrf.2130

ISSN : 1952-403X

Éditeur :

Armand Colin, Société des études robespierristes

\section{Édition imprimée}

Date de publication : 1 mars 2005

Pagination : 79-97

ISSN : 0003-4436

\section{Référence électronique}

Luc Nemeth, « Un état-civil chargé d'enjeux : Saint-George, 1745-1799 », Annales historiques de la

Révolution française [En ligne], 339 | janvier-mars 2005, mis en ligne le 15 mars 2008, consulté le 22 avril 2022. URL : http://journals.openedition.org/ahrf/2130 ; DOI : https://doi.org/10.4000/ahrf.2130

Ce document a été généré automatiquement le 22 avril 2022.

Tous droits réservés 


\title{
Un état-civil chargé d'enjeux : Saint- George, 1745-1799
}

\author{
Luc Nemeth
}

Un terrain, propice à l'erreur

1 Une brève, parue dans le Journal de Paris du 26 prairial an VII (14 juin 1799), signale que «Saint-Georges, célèbre par sa supériorité dans les armes, la danse, l'équitation, la musique, est mort à Paris, rue Boucherat, le 21 prairial, à l'âge de 60 ans " ${ }^{1}$. Et... point final. C'est que déjà le célèbre mulâtre incarnait, aux yeux de son époque, un passé éloigné. Pourtant, pas plus tard qu'en 1796, il participait encore aux combats qui se déroulaient à Saint-Domingue, et le bruit de sa mort avait même circulé ${ }^{2}$; sitôt rentré à Paris il avait repris la direction de concerts, dont la presse n'avait pu que saluer la "supériorité de l'éxécution " ${ }^{3}$; le temps n'était pas si loin où en 1793 , alors qu'il dirigeait un corps d'armée à Lille, il avait joué un rôle non négligeable, au moment de la trahison de Dumouriez ${ }^{4}$; dès 1790 , alors que la Constituante se refusait à abolir l'esclavage, le lobby des planteurs coloniaux, mieux connu sous le nom de Club Massiac, avait pu voir en lui un adversaire irréductible ${ }^{5}$; à la veille encore de la Révolution, pour peu qu'il jouât du violon dans ce haut lieu de plaisir populaire qu'étaient les jardins du Palais-Royal, « la foule s'y portait " ${ }^{6}$, tout comme le fit après juillet 1790 la population lilloise aux concerts, aux représentations d'opéra ou aux duels (d'exhibition) qu'organisait ce capitaine de la garde nationale ${ }^{7}$. Mais lui aussi subissait maintenant la dure loi : pour avoir trop bien incarné l'avenir, il ne pouvait plus être identifié même lointainement au présent...

Passé l'annonce de sa mort l'oubli continua et se vit même conforté, trois ans plus tard, avec le rétablissement de l'esclavage : cette décision (du 20 floréal an X - 10 mai 1802) impliquait de voir effacé de l'imagerie officielle tout homme de couleur ayant pu jouer quelque rôle dans la période récente. Quant à l'abolition ultérieure de 1848, dans la mesure où elle ne s'attaquait pas au statut colonial, elle impliquait le maintien à l'arrière-plan de tous ceux des colonisés qui jadis avaient risqué leur vie pour la Nation, dans une perspective égalitaire implicite. 
3 En toute équité, il convient d'ajouter que de son vivant déjà Saint-George n'avait pas été bien traité. À peine avait-il pris en septembre 1792 le commandement d'une légion de volontaires de couleur, "la Légion des Américains et du Midy», ou "Légion Noire», ou "Légion Saint-George " ${ }^{8}$, que l'on avait banalisé celle-ci, devenue le "13e régiment de Chasseurs à cheval» (la faille, ne fut pas bien difficile à trouver : en langage militaire une légion se compose d'un corps de Cavalerie et d'un corps d'Infanterie; aussi on s'aperçut... trois mois plus tard, que seul avait été déclaré le corps de Cavalerie [ $\left.{ }^{\circ}\right]$ ). Son rôle au moment de la trahison de Dumouriez ne l'avait pas empêché d'être détenu au château (aujourd'hui démoli) de Hondainville, près de Clermont de l'Oise, et ce, pour la plus mauvaise raison : on lui reprochait d'avoir fait partie du clan Philippe-Égalité, Dumouriez, etc., là où précisément il n'avait pas hésité à se séparer d'eux, par fidélité envers la Révolution. Certes, il avait fini par être libéré au bout de dix-huit mois ${ }^{10}$, grâce au 9 Thermidor, mais il n'avait jamais pu obtenir d'être rétabli dans son commandement; et c'est dans un relatif dénuement qu'il était mort chez Nicolas Duhamel ${ }^{11}$, un de ses lieutenants de l'ex-légion, chez qui il était maintenant hébergé.

4 Une vingtaine d'années plus tard, quiconque aurait voulu en savoir un peu plus le concernant ne pouvait que se reporter aux mémorialistes d'époque ou à des nécrologies, fort pertinentes à bien des égards ${ }^{12}$, mais qui en ce qui concerne la date de naissance par exemple étaient soit muettes, soit erronées. En effet, à sa mort il était âgé de cinquante-trois ans et non de "soixante », comme ce fut imprimé on l'a vu - et avec une conséquence imprévue : l'officier d'état-civil, qui était resté muet sur ce point faute d'en savoir plus, rajouta quelques jours plus tard sur le registre des décès, croyant bien faire, l'indication "soixante ans »; et c'est ainsi que l'année 1739 a pu être avancée comme date de naissance; en 1972 encore, Odet Denys, dans ce qui constitue la première véritable biographie, pourra seulement écrire que Saint-George était né " entre les années 1739 et 1745 " $^{13}$ (l'état-civil ancien n'étant plus là, pour arbitrer).

5 Pour encore un peu plus compliquer les choses : la seule donnée d'état-civil qui puisse être attribuée à Saint-George lui-même, est fausse! Disons qu'il avait menti, pour la bonne cause : alors qu'il servait dans la garde nationale en 1791, il avait eu à préciser son âge et avait indiqué « quarante-deux ans ${ }^{14}$; ce qui le faisait naitre en 1748-1749 (au lieu de 1745). Bien entendu une coquetterie est toujours possible, mais le bénéfice résultant - trois ans - paraît si mince, qu'il faut peut-être envisager autre chose. À cette date, le «modèle » omniprésent était celui constitué par la République romaine - où était mobilisable tout homme valide, âgé de dix-sept à quarante-six ans. Certes, le contenu des registres atteste qu'à Lille on regarda peu à l'âge, pour accepter ceux qui se proposaient. Mais nul ne pouvait préjuger de l'avenir. On ne peut exclure qu'il aît considéré que c'était "aussi bien", et même mieux, de procéder ainsi.

Enfin, il n'y avait pas de descendants pour fournir ce genre de précisions. Sa demiesœur, née du même père et de l'épouse légitime de celui-ci, ne fut pas élevée avec lui ${ }^{15}$. Seuls deux d'entre ceux qui l'avaient connu dans le milieu des salles d'armes (à Paris ou à Londres) venaient de lui consacrer chacun quelques pages, dans un de leurs ouvrages. La Boëssière fils, en 1818, rappelait qu'il était né le 25 décembre 1745 ; Henry Angelo, un an plus tôt, avait indiqué que " c'était le fils de M. de Boulogne, un riche planteur de la colonie ${ }^{16}$. Indications exactes, l'une comme l'autre, mais dont la seconde cadrait mal avec les grandes lignes de l'histoire officielle, sous la Restauration. Certes, un planteur pouvait avoir eu ses entrées à la Cour, mais tout cela sentait un peu le nouveau riche. De plus, ce n'est pas tant du père qu'il s'agissait, que du fils : un mulâtre ayant eu 
du talent - et pour le violon, qui plus est - ne pouvait qu'avoir été le fils, même illégitime, d'un aristocrate! Encore, s'il s'était contenté de jouer du violon : on aurait pu lui attribuer des... dons d'imitation - théorie alors très à la mode, à propos des hommes de couleur. Mais là, ce n'était plus possible : il avait composé pour l'instrument!

Une notice biographique aventureuse

7 La Biographie universelle, qui faisait alors autorité, rendit son oracle, en 1825 : «le chevalier fut amené fort jeune en France par M. de Boulogne, fermier-général, son père, qui l'avait eu d'une négresse ${ }^{17}$. Va pour « chevalier ", titre qui n'était plus guère attribué que pour de hauts faits, et dont il y a peu de chances que Joseph aît été pourvu à son arrivée en France. Va pour " négresse ", terme qui donnait à cette notice un air de sincérité - et ce alors même que l'on venait d'égarer le lecteur, avec l'entrée en scène d'un «fermier-général ».

8 L'erreur put ensuite se déployer, prenant même un aspect érudit, avec des variations sur les titres et prénoms du père : tantôt il s'agissait de "Jean-Nicolas de Boulongne, contrôleur général des finances » - telle est notamment la paternité reprise en 1875 par Pierre Larousse ${ }^{18}$, dans sa solide notice; tantôt, du fermier-général Philippe-Guillaume de Boullongne-Tavernier; tantôt encore du frère du précédent, Pierre-Guillaume Tavernier de Boullongne ${ }^{19}$. L'important étant bien sûr qu'à son arrivée en métropole, le petit aît pu habiter un hôtel particulier. De plus, avec le patronyme " de Boulongne » et ses dérivés, une avantageuse hérédité artistique en découlait pour lui, puisqu'il devenait de fait le parent d'une famille de grands peintres. Enfin, la société du dixneuvième, s'il lui fallait bien composer avec la relation dite inter-raciale, préfèrait qu'à tout prendre elle concerne (ou ici aît concerné) des personnes de même rang : attribuer à Saint-George une origine aristocratique était aussi, consciemment ou non, un moyen d'excuser (!) des femmes de très haut rang, dont on assure que toutes n'avaient pas été insensibles au charme de ce mulâtre... ${ }^{20}$.

Le plus piquant est qu'ici les tenants de l'hérédité prestigieuse, comme c'est souvent le cas, allaient chercher fort loin, ce qui eût été à portée de leur main. Car, si le père est le planteur Georges de Bologne Saint-Georges, alors le petit aura eu pour oncle naturel le frère de celui-ci, Pierre de Bologne, personnage que les archives décrivent comme portant perruque ${ }^{21}$, mais qui surtout fut en son temps un poète reconnu ${ }^{22}$; né en Martinique, il vivait alors en métropole, mais avait conservé des intérêts aux "Isles ", et "partageait son temps entre l'Angoumois et les Antilles » ${ }^{23}$. Ajoutons que Georges de Bologne lui-même, si ses revers de fortune l'amenèrent devant les tribunaux ${ }^{24}$, et si sa particule n'est pas mieux assurée que celle de son frère, n'était pas non plus le dernier venu; et il obtiendra, en 1757 , le brevet de "gentilhomme ordinaire du roi ${ }^{25}$. On comprend mieux du même coup la facilité avec laquelle, se voyant menacé d'être puni de mort suite à un duel qui s'était terminé tragiquement - son adversaire, qui n'était qu'égratigné en apparence, décéda peu après -, il obtint en métropole, où il était allé les chercher, ses "lettres de rémission ». Par ailleurs lui-même, tout comme son frère, avait fait l'apprentissage des armes dans une des compagnies de mousquetaires ${ }^{26}$ (privilège en principe réservé aux jeunes gens "bien nés", mais qu'une fortune

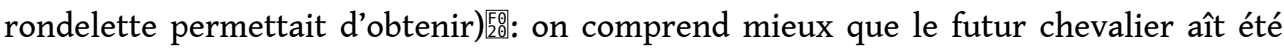
admis pour apprentissage chez le fameux maître d'armes La Boëssière ${ }^{27}$ ou encore qu'en 1764, à l'issue de cette formation, il aît été admis dans le corps des Gendarmes de la garde ${ }^{28}$. 
10 En tout cas, une chose est sûre : ce n'est pas Saint-George qui est à l'origine du glissement des dictionnaires en direction de paternités aristocratiques. On a pu voir (cf. témoignage d'Henry Angelo, supra) qu'il ne se cachait pas d'être fils d'un planteur. Par ailleurs, le littérateur Moline, qui fut plus tard secrétaire-greffier à la Convention, composa un jour un compliment destiné à être gravé sous le portrait du musicien ; il l'adressa au Mercure de France, qui le publia, sous le titre : "Vers au bas du portrait de M. Boulogne de Saint-George ${ }^{29}$; or, si les Boulogne ou Bologne étaient nombreux (les deux orthographes étant équivalentes), et s'il y eut plusieurs chevaliers de SaintGeorges ${ }^{30}$ : il n'y eut jamais, à notre connaissance, qu'une seule famille Boulogne de Saint-Georges. Et dans le cas précis, il ne s'agissait pas du père...

Un "roman" très louis-philippard

11 En 1840 un polygraphe alors en vogue, et à propos duquel Alexandre Dumas aura eu un mot cruel pour expliquer qu'il n'aît pas atteint la gloire («Roger de Beauvoir eut, au commencement de sa carrière, un grand malheur. Seul d'entre nous, il était né riche [31]), publia deux œuvres de même titre, Le Chevalier de Saint-Georges. L'une, écrite en commun avec Mélesville, le librettiste de Scribe, était une pièce de théâtre, " comédie mêlée de chant ${ }^{32}$, dont la première eut lieu au Théâtre des Variétés le 15 février 1840 ; l'autre, un roman ${ }^{33}$. La pièce est bien enlevée et, si elle ne fustige ni le colonialisme ni l'esclavage - la censure, qui n'était nullement débonnaire, ne l'eût pas permis -, elle ridiculise assez efficacement, sinon le préjugé de couleur dans son ensemble, du moins l'attitude de ceux que l'on appelle familièrement des « racistes ». Le roman, lui, cumule les maladresses - à trop vouloir concilier des impératifs contradictoires.

D'un côté, l'auteur est tenu de porter un regard favorable sur Saint-George, pour une raison qui va plus loin que les règles du genre : le roi, Louis-Philippe, avait dans sa prime jeunesse côtoyé le héros, dans cette mouvance qui gravitait autour de PhilippeÉgalité et Dumouriez; or le roi ne saurait qu'avoir eu toujours de bonnes fréquentations! Mettre en avant un tel personnage, sous l'angle du " patriotisme », est même tout à fait compatible avec l'image de roi-patriote, qui est maintenant à l'ordre du jour. De plus, le contenu du roman, même si l'auteur n'est pas toujours très cohérent dans son rejet du préjugé, et qu'il reprend divers poncifs de la littérature exotique, confirme qu'il n'avait que mépris pour les comportements racistes.

13 Mais, d'un autre côté, ce roman tente de ménager un public qui est non seulement bourgeois, mais monarchiste. Avec «bourgeois ", pas de problème : cette classe est déjà en partie résignée à l'abandon de l'esclavage, si c'est là le seul moyen de conserver ses colonies ${ }^{34}$; celles-ci ne sont pas encore objet de la même charge émotionnelle que sous la Troisième République ; le lobby colonial, dont les sollicitations constantes suscitaient déjà quelque agacement, ne pèse plus du même poids, depuis que la betterave sucrière est venue mettre fin au monopole de la canne à sucre ; et si la censure veille encore, et impose de faire preuve de prudence pour aborder tous ces thèmes, Victor Hugo a ouvert une brèche avec Bug-Jargal en 1826. Mais pour ce qui est de "monarchiste ", c'est autre chose : le lecteur risque de voir un affreux traître en ce Saint-George, qui non seulement s'est acoquiné à Philippe-Égalité, cousin régicide, mais qui, ayant compris l'impossibilité en France d'une solution "à l'anglaise", a laissé de côté les réserves que lui inspirait l'aile agissante de la Révolution : réserves qu'en 1790, et bien qu'ayant rejoint la garde nationale à Lille, il avait exposées dans une brochure ironiquement titrée Nous sommes donc trois ? ${ }^{35}$; brochure dans laquelle il se posait en partisan du «juste milieu ». Aussi, de Beauvoir s'en sort-il en faisant endosser à Philippe- 
Égalité le «mauvais » rôle, et en se livrant à un pieux mensonge : oui bien sûr, SaintGeorge a rejoint ce débauché, mais... c'était juste pour l'accompagner dans ses virées nocturnes! Rien ne nous est épargné de l'amour que, en secret, Saint-George est supposé avoir voué à la reine. Et on ne s'étonnera pas de voir le roman se terminer au... début des années 1790 , c'est-à-dire d'une certaine manière, au moment où "tout" commence.

Ajoutons qu'en 1840, lorsque parait ce livre, la plupart des témoins ne sont plus là pour apporter une précision, ou un éventuel démenti. La légende, avec ses clichés, a eu le temps de recouvrir celui que de son vivant déjà on appelait «le fameux Saint-George » ${ }^{36}$, qui par la suite aura eu droit jusqu'au surnom de "Don Juan noir ", qui à sa mort avait pu être présenté comme le " Voltaire des arts d'agrément » ${ }^{37}$ (l'abbé Grégoire parlera plus tard de "Voltaire de l'équitation, de l'escrime, de la musique instrumentale » [38]), et qui amena Balzac à écrire, venu le moment de brosser le portrait de son personnage Victurnien d'Esgrignon : "il faisait des armes comme un Saint-George» ${ }^{39}$. L'auteur luimême, qui admet n'avoir pas voulu faire œuvre exclusive de biographe, mais de romancier ${ }^{40}$ - on sait toutefois qu'il ne suffit pas que le procédé s'avoue, pour cesser de produire ses effets -, alterne allègrement le vrai et le faux. C'est ainsi que l'enfance du chevalier est décrite comme s'étant déroulée à Saint-Domingue, là où rien ne prouve qu'il aît seulement séjourné ${ }^{41}$ : le but est ici de flatter l'imaginaire colonial d'une société pour laquelle Saint-Domingue reste "la" grande île - et Martinique et Guadeloupe, de simples extensions. Pour bien marquer qu'il s'agit d'une fiction, il dénomme la mère Noëmi, alors qu'elle s'appelait Nanon. Peu de changement, en revanche, pour le père, qui s'il n'est plus fermier-général, reste un notable de passage : « Monsieur de Bollogne, contrôleur général » ${ }^{42}$.

15 C'eût été faire un grand honneur à ce roman, qui se voulait surtout distrayant, que d'aller en dénoncer une erreur de généalogie ! D'ailleurs, on voit mal qui aurait pu avoir envie de le faire : sûrement pas, en tout cas, ceux qu'animait l'esprit de justice. Car enfin le vrai père, le sucrier et caféier Georges de Bologne ${ }^{43}$, symbolisait l'Ancien Régime sous sa pire forme, qui persistait : l'esclavage. La plantation Bologne avait été soumise au même régime que les autres, et ce dans ces années qui en Guadeloupe sont des années de répression accrue ${ }^{44}$. De plus, s'il avait fait élever "comme s'il avait été le sien ", l'enfant qu'il avait eu d'une esclave, on ne pouvait, dans le contexte, invoquer le seul physique de Nanon, même s'il fut peut-être hors du commun (cf. Henry Angelo : "she was justly considered as one of the finest women that Africa had ever sent to the plantations " [ $\left.{ }^{45}\right]$ ). Dès lors, on était a priori en présence d'un acte de générosité : il y avait, on l'admettra, d'autres priorités que d'aller faire de la publicité à ces rares cas, alors même que l'abolition restait un combat à mener, et dont le résultat n'était aucunement acquis.

Mais ce fut bien au-delà de l'état-civil, que ce roman hypothèqua l'avenir. Saint-George venait d'être présenté comme un héros de cape et d'épée : quel historien aurait voulu en savoir plus sur un tel personnage? Aussi, ce roman resta la référence obligée jusqu'à ce qu'en 1923 le musicologue Lionel de La Laurencie ne consacre au virtuose et compositeur une étude qui est un modèle du genre, dans un des volumes de son École française de violon ${ }^{46}$. Bien qu'une phrase qui s'y trouve aît pu choquer plus d'un lecteur ( «L chevalier de Saint-Georges était, en dépit de son teint, un tendre, un sentimental » $\left.\left[{ }^{47}\right]\right)$, on peut estimer qu'avec cette cinquantaine de pages, qui s'appuient à la fois sur une parfaite connaissance de l'œuvre, et celle d'archives militaires ici indispensables ${ }^{48}$, 
la recherche répond enfin aux critères habituellement exigés. Mais, concernant la paternité, La Laurencie en reste à ce qui avait été énoncé dans le roman, quatre-vingt trois ans plus tôt : «M. de Boulogne, contrôleur général " ${ }^{49}$. La caution apportée à une erreur par un grand nom de la musicologie assurait à celle-ci un bel avenir.

La vérité, assez tardivement

Personne apparemment ne semble avoir noté cette bizarrerie : sur une étude de cinquante-deux pages un musicologue venait d'en consacrer quarante-et-une (!) à faire un travail que les historiens n'avaient pas eu envie de faire; seules les onze dernières traitaient de musicologie...

Mais on ne saurait tenir un discours aussi sévère à propos de la Guadeloupe, où avait toujours existé un intérêt pour ce personnage ; même les enfants des écoles savaient qui il était, car des manuels scolaires à usage local le mentionnaient; et, même si les initiatives officielles sont à interpréter avec prudence, on peut noter qu'en 1912 le Conseil municipal de Basse-Terre décida d'attribuer son nom à une rue ${ }^{50}$. Mais les érudits, eux, n'eurent jamais la tâche facile, vu la difficulté à se procurer les ouvrages nécessaires. Ainsi Gaston Bourgeois (Chef du Service de l'Enregistrement à BasseTerre), qui dans les années trente entreprit cette biographie, dût-il se mettre en contact avec un service de documentation qui, de Paris, lui envoyait des photocopies! Ses courriers de remerciement attestent ses résultats; en mars 1935, il écrit : «les très intéressants renseignements que vous me donnez sur les Boullongne confirment ma thèse dont je puis, d'ailleurs, démontrer l'exactitude : le Chevalier n'est pas le fils du Contrôleur général des Finances, ou du Fermier Général il est fils d'un colon " ${ }^{51}$. Qui saura jamais ce qui le fit changer d'avis et publier en 1964, soit vingt-neuf ans plus tard, un bref article dans lequel il reprenait une " paternité » erronée ${ }^{52}$. Pourtant, on peut estimer qu'en dépit d'erreurs qui n'épargnaient pas non plus la date de naissance, cet article eut le mérite de relancer ces recherches.

Les historiens guadeloupéens enfin ne disposaient pas des mêmes facilités éditoriales, que leurs homologues du vieux continent : ainsi Vauchelet, qui en 1906 avait écrit cette biographie, dût-il se contenter d'en publier des extraits dans le Messager de la Guadeloupe ${ }^{53}$. Finalement c'est un originaire de l'île, Odet Denys, qui en 1972 publiera la première biographie ${ }^{54}$; mais, en dépit de ses relations (il avait fait carrière d'avocat en métropole), il lui faudra s'adresser à une maison d'édition militante, ce qui condamnait l'ouvrage à une diffusion limitée. L'objectif était modeste : il s'agissait surtout, dans une perspective de vulgarisation, de faire échapper à l'oubli, celui qui était peu à peu devenu un "inconnu de l'Histoire" - il n'y a donc pas à appliquer ici les critères stricts de la recherche, comme le fit non sans quelque déloyauté une revue savante ${ }^{55}$. Vu les priorités qui sont les siennes, il cite ses sources, mais il le fait en fin d'ouvrage, et sans appel de notes. Bien entendu il donne l'identité du père, et il est même le premier à le faire en toute exactitude (« Monsieur de Bologne Saint-Georges» $\left.{ }^{56}\right]$ ) ; mais il le fait incidemment et semble avoir été persuadé que le prénom était un renseignement qui ne pouvait intéresser le public : pourtant on a la preuve qu'il le connaissait puisque parmi ses notes en fin de texte figure la cote d'un acte officiel, nominatif ${ }^{57}$.

On est devant un autre type d'ouvrage avec la biographie de référence, qui a été publiée en 1996 en Suisse, par le musicologue Emil F. Smidak. Le souci de la précision transparaît jusque dans le titre : Joseph Boulogne nommé Chevalier de Saint-Georges ${ }^{58}$. La volonté de faire œuvre d'historien apparaît dès la première partie, qui est autant d'utiles rappels sur l'esclavage, la traite, l'histoire de l'Afrique ancienne. Les normes de 
la recherche la plus exigeante sont ici respectées. De plus l'auteur, outre qu'il semble avoir brassé une imposante quantité d'archives, en extrait le contenu avec talent. Ainsi note-t-il que dans un mémoire datant de 1782, et consacré à un litige de sépulture, le curé d'une paroisse de Basse-Terre attribue deux enfants, au défunt Georges de Bologne : or celui-ci n'a eu qu'un seul enfant déclaré ; la probabilité d'un enfant illégitime s'en trouve déjà confortée... ${ }^{59}$. Par ailleurs, sa maitrise des sources lui permet d'affirmer qu'aucun des "pères" avancés précédemment, mis à part celui identifié par Odet Denys, n'avait les attaches guadeloupéennes requises. Et cette fois ce n'est plus une incidente, mais tout un chapitre, qui vient d'être consacré à ce vrai père.

Une paternité cousino-linéaire?

21 En 1999, a été commercialisé un ouvrage intitulé Monsieur de Saint-George (on entrevoit déjà le public ciblé) ; pour l'essentiel ce n'est qu'une besogneuse compilation, mais on y trouve aussi quelques scènes originales, et même très originales, comme celle-ci, qui est supposée se passer à Paris, après que le futur virtuose aît quitté à jamais son pays natal :

«Entre les multiples enseignements des précepteurs et les promenades parisiennes avec les gouvernantes, la vie pour le métis de la place des Victoires a fini par tomber dans une confortable monotonie lorsqu'un jour du printemps 1749, au retour d'une promenade dans les rues de Paris, il est happé par deux bras puissants qui le projettent en l'air : "Enfin le voilà mon petit chevalier, mon chevalier de Saint-George! Mais c'est que tu es devenu un vrai petit homme!" Le personnage tonitruant à la force herculéenne n'est autre que le "cousin" Georges Bologne de Saint-Georges $[. ..]{ }^{60}$

Pour sûr, on veut bien croire que le tonitruant personnage aît pris le petit dans ses bras, puisque sauf cécité des bons auteurs l'intéressé n'était pas le « cousin »-va pour les guillemets -, mais, le père! Sachant que, par ailleurs, l'auteur ne peut prétendre qu'il n'a pas lu ses prédécesseurs, ce n'est pas sans raisons qu'il s'est exposé à l'accusation de... substitution paternelle.

Une longue parenthèse sera ici nécessaire pour évoquer une histoire déjà connue de certains lecteurs, et qui commence au début du XvII ${ }^{e}$ siècle. Cette histoire est celle d'un parcours collectif : celui de protestants français persécutés qui allèrent au Refuge comme on disait alors, à propos de Rotterdam. De là, une partie d'entre eux repartit pour le Brésil, à Pernambouc (actuelle Récife). En 1654 la couronne du Portugal, après plusieurs années de conflit, parvint à recouvrer sa pleine souveraineté sur la région, et les expulsa. Au nombre d'environ neuf cents, ils tentèrent de débarquer en Martinique, mais le gouverneur, sous la pression des jésuites, les refusa ; puis, ils s'installèrent en Guadeloupe ${ }^{61}$. Après la révocation de l'Édit de Nantes en 1685 , ils allèrent vers les Antilles anglaises ou hollandaises, où la religion était libre. Mais en général ils laissaient sur place un membre de la famille qui simulait la conversion, en attendant le moment où la revente des biens redeviendrait possible : ce moment n'arriva pas, et, à force de simuler, ce furent eux qui finirent par être assimilés ${ }^{62}$. Ce processus, toutefois, fut assez lent. Les jésuites ne manquent pas de se plaindre, dans leurs courriers, du peu de zèle de ces convertis : il n'était pas rare de les voir abjurer plusieurs fois de suite et, pour tout dire, redevenir catholiques, lorsqu'ils avaient besoin du curé. L'assimilation s'accentua malgré tout, au début du siècle suivant. Un document de 1711, intitulé Liste des Religionnaires Et Nouveaux Convertis qui sont aux Isles françoifes de l'Amérique, nous dit que «la Veuve Bologne ne fait aucun Exercice de Catolicité, mais Ses Enfans font les devoirs » ${ }^{63}$. Mais, en 1740 encore, on peut noter que le père, Georges de Bologne, n'est 
toujours pas représenté au baptême de sa fille ${ }^{64}$ : c'était là un moyen courant de rappeler l'ancienne foi. Cela n'empêcha pas ces protestants de se comporter comme les autres esclavagistes ; mais, lorsqu'on se trouve face à des comportements atypiques, ce n'est pas inutile de savoir que subsistait, dans ces familles, "une mentalité d'indépendance vis-à-vis du pouvoir ${ }^{65}$.

Il est certain qu'ainsi contée l'histoire familiale n'est pas moins intéressante, sans qu'il ne faille y chercher des explications mécanistes : même ce père n'aurait sans doute pu dire si c'était le souvenir de la persécution qui le fit prendre en charge l'enfant d'une esclave ou encore le fit, lorsqu'il alla chercher sa grâce en métropole (cf. épisode du duel tragique), emmener avec lui Nanon et son fils, craignant de les voir vendus en son absence en cas de saisie de ses biens ${ }^{66}$. Mais, quelle que puisse être la réponse, on est ici devant le genre d'interrogation qui conditionne la "carrière" d'un ouvrage, commercialement parlant. Car le lecteur, sitôt qu'il l'a en tête, ne peut plus s'en défaire, au point qu'elle finit par fonctionner comme une grille de lecture. Bref, on est ici devant une autre histoire, et qui est en tout état de cause fort éloignée du public visé par Monsieur de Saint-George...

Odet Denys et Smidak s'étaient-ils trompés ? À défaut de "piste" de vérification, nous sommes partis d'un courrier du 5 novembre 1749, consécutif à cette affaire de duel, et signalant que le sieur Bologne vient d'obtenir ses lettres de rémission. Ce courrier ne s'en tenait pas là : il y était précisé que l'intéressé "repasse aux Isles » ${ }^{67}$ : qui sait, avec un peu de chance, sur une liste de passagers... Disons que nous avons été particulièrement servis par la chance. En effet, nous n'avons consulté que les listes de Bordeaux, qui apparaissait comme le port d'embarquement le plus plausible (mais en pure hypothèse); d'autre part, cette lettre est du 5 novembre, mais nous avons pensé que le contenu était déjà peut-être un peu daté et que mieux valait commencer la vérification deux ou trois mois plus tôt; or, c'est à la date du 3 septembre qu'apparaissent, parmi les passagers en partance ${ }^{68}$, à destination de la Martinique :

Il n'y a pas là de révélation, donc, mais la confirmation du "profil" de planteur créole jadis indiqué par Angelo, ainsi que de l'identité établie en 1972 par Odet Denys ; quant à la Martinique, ce détour était ici obligé - le Conseil Souverain de cette île était compétent en appel pour les jugements rendus par le Conseil Supérieur de la Guadeloupe.

Le nom de ce père et celui du fils figurent également sur un registre de passagers à l'arrivée ${ }^{69}$, en provenance de Guadeloupe cette fois-ci, le 12 août 1753 :

Les demoiselles Merican sont ici l'épouse légitime du père, qui ne saurait prétendre au titre de « dame », et sa fille, la demie-sœur de Joseph ; «J.h » est l'abréviation courante de ce prénom; le père utilise volontiers, en voyage, le nom «Saint-Georges » pour se différencier de son frère.

31 Enfin cette vérification, qui avait pour seul but d'obtenir confirmation de l'identité paternelle, a permis de dissiper le doute qui entourait encore la date de naissance. Les 
indications ci-dessus proviennent de registres, mais les pièces d'origine ont été conservées, pour l'année 1749 ; ces « rôles d'équipage » étaient établis avec grand soin, mentionnant jusqu'à la moindre substance de la pharmacie de bord, et, pour les passagers, leur âge. Ainsi, nous apprenons qu'à bord de l'Achille avaient embarqué ${ }^{70}$, ce 3 septembre :

«Le Sr George Bologne habitant de la Guadeloupe agé de 38.- ans

La nommée Nanon Negreffe agée de 26. ans appartenante au dt Sr Bologne

Le nommé Joseph son fils agé de 3 ans »

Ici encore il s'agit d'une confirmation : la date de naissance 1745 avait été indiquée par La Boëssière fils, en 1818 ; probablement l'avait-il trouvée dans les dossiers de son père. Ces archives suggèrent, par ailleurs, une configuration familiale hors du commun. En effet, ce n'est pas le 3 septembre mais le 1er août que le père était supposé embarquer, et en compagnie de son épouse légitime ; or seule celle-ci embarqua, ce jour-là, tandis que le père repartit quelques semaines plus tard, en compagnie de Nanon ${ }^{71}$.

État-civil et identité historique

La brève qui, dans le Journal de Paris du 14 juin 1799, avait annoncé la mort de SaintGeorge (cf. supra), ne manquait pas de roublardise. Car, en dépit de sa brièveté, elle parvenait à donner l'illusion du trop-plein, avec son allusion à la supériorité de celui-ci dans «les armes, la danse, l'équitation, la musique»! Bref, on continuait de lui reconnaître tous les talents, pourvu qu'ils fussent limités aux domaines sportif et artistique... Les dictionnaires et encyclopédies n'eurent plus qu'à emboîter le pas, et bientôt ce devint un tic de plume que de dire de lui, en reprenant (hors contexte) une formule de l'abbé Grégoire, qu'il " excellait dans les arts d'agrément » ${ }^{72}$.

La chute de la monarchie de Juillet, puis celle du Second Empire, ne permirent pas de voir ce personnage enfin exposé dans la perspective historique : une telle évocation, en effet, aurait eu à s'attarder sur l'épisode de la «légion noire». Or l'idée qu'aient pu exister en 1792 des "volontaires" de couleur, même s'ils ne furent que quelques centaines - ce qui n'est pas négligeable, compte tenu du faible effectif de cette population en métropole -, était une idée qui dérangeait. Elle éveillait un sentiment de culpabilité, vu le rétablissement de l'esclavage par Bonaparte dix ans plus tard. Elle allait à l'encontre de l'interprétation jacobine admise. Elle ne pouvait pas même se prêter à une récupération de type exclusivement "patriotique ", puisque ces hommes avaient aussi été partie prenante dans le combat visant à abolir l'esclavage ${ }^{73}$. Enfin, par le caractère décidé de leur démarche ${ }^{74}$, ils s'étaient placés d'emblée en dehors de tout antiracisme ne reposant que sur la charité.

37 À ce tabou (très spécifique) s'ajouta le total désintérêt du xix ${ }^{e}$ siècle, comme pouvait déjà le noter en son temps Claude Perroud ${ }^{75}$, pour tout ce qui avait concerné la Société des Amis des Noirs, et les éléments ayant gravité autour de celle-ci : or Saint-George aura eu tout loisir d'être en contact avec les fondateurs anglais, en 1786-1787, durant son premier séjour à Londres ${ }^{76}$. Et, même si ce peut avoir été sans objet, une des grandes craintes du Club Massiac, au début de l'année 1790, est de le voir en train de préparer (avec d'autres) l'expédition qui pourrait tenter le débarquement à SaintDomingue ${ }^{77}$. En tout cas il aura porté lui aussi, dans les milieux académiques, le poids de ce manque d'intérêt.

38 Lorsqu'arriva le temps de l'école publique, et que les manuels scolaires composèrent l'album de famille républicain, la récupération à laquelle on pouvait éventuellement 
s'attendre n'eut pas lieu. Peut-être aura-t-on intuitivement compris la difficulté qu'il y aurait eu à le présenter comme étant venu se mettre "au service» (sic) de la Révolution...

Il aura donc fallu attendre 1923, soit cent vingt-quatre ans après la mort de Joseph Boulogne dit Saint-George, pour qu'une étude documentée lui soit consacrée ; encore ne le fut-elle que dans le cadre de recherches spécialisées de musicologie, qui avaient peu de chances de recueillir une large audience, et ne stimulèrent pas la curiosité des historiens. Peut-être même, interpelés, auraient-ils fait valoir que c'était à des collègues antillais que revenait d'étudier ce personnage... Quoi qu'il en soit, il y avait bien eu occultation, et qui s'était en grande partie jouée autour de cet épisode de la "légion noire». La facilité avec laquelle aura pu ensuite s'installer, puis perdurer, un état-civil erroné, n'en fut jamais qu'une des banales conséquences.

On ne saurait enfin perdre de vue que l'état-civil n'est que l'un des aspects de la généalogie. Qu'il me soit permis de citer la lettre que Saint-George adressa aux autorités lilloises le 4 mai 1793, soit deux jours après que le pouvoir exécutif aît commencé à lui chercher querelle :

«-Lille le 4 may 1793 l'an deux

de le République française.

Citoyens officiers municipaux

Je viens de recevoir une lettre du ministre aupres duquel on cherche a nuire a mon corps, qui comme vous le savez, a toujours fait le service tout seul dans les decouvertes, cantonnements, detachements, avant garde et escorte de vivre et de munitions avec la plus grande exactitude, zele, et patriotisme. C'est d'apres cette verité qui doit vous etre connue et mon devouement aux citoyens lillois avec qui j'ay servi dans la garde nationale, que je reclame votre justice pour eviter a l'avenir la persecution des ames mal intentionnés. Pour le service de la Republique, ma vie et mes talents sont consacrés pour mes freres, et rien dans le monde ne pourra alterer mes sentiments patriotiques et republicains.

St George -colonel du 13me Regt de chasseurs» ${ }^{78}$

41 On a bien lu : « mes frères "... Il peut ne s'être agi que de la fraternité d'armes, mais on a pu lire que cette unité procèdait à un recrutement ethnique. Voulait-il faire allusion à cela? L'important alors, et plus que jamais, serait moins le nom du père qu'une mère dont nous ne connaissons guère que le prénom, attribué "au nom du Père ». Si, par ailleurs, la composante ethnique ne peut se réduire à l'influence maternelle, il nous faut bien signaler qu'en 1755, la même année que celle où l'enfant (âgé de dix ans) fut envoyé en métropole ${ }^{79}$, la mère, dans le contexte particulier déjà signalé, débarqua à Bordeaux avec le père ${ }^{80}$; et elle resta plusieurs années au bas mot en France puisqu'en 1762 elle alla se déclarer au greffe de l'Amirauté ${ }^{81}$, lorsque cette formalité devint obligatoire même pour les « libres ».

Qu'il nous faille insister sur cette composante en dit long sur une insidieuse présentation, qui fait surface de temps à autre, et qui vise à faire de ce mulâtre le "premier colonel noir de l'armée française ». C'est là, d'abord, une fanfaronnade quelque peu déplacée, car s'il est une chose dont l'administration française peut bien rougir c'est la façon dont fut traité ce gradé, qui, lorsqu'il fut remis en liberté en 1795, ne retrouva pas même le commandement de son régiment, ou d'un autre. Mais faire abstraction de ce qu'il aura pu subir dans sa carrière, c'est aussi se priver d'un élément de compréhension important si on espère saisir ce qui fit que ce personnage, qui apparaît a priori comme un "modéré" de la Révolution, opta pour une position de défense intransigeante de celle-ci, quitte à désavouer un Dumouriez dont il était proche 
(on trouva même, lors d'une perquisition effectuée dans la maison qu'il occupait à Lille, une pièce cadenassée contenant des effets ayant appartenu à Philippe-Égalité et Dumouriez ${ }^{\left[{ }^{82}\right]}$ ) : tout ce qui équivalait à un retour - même partiel - de l'ordre ancien, était pour lui un affront.

Une commémoration très parisienne

Pour l'essentiel, l'existence de Saint-George se déroula en métropole. Il put y cultiver certains de ses dons, plus aisément qu'il n'aurait pu le faire dans les "Isles ", et côtoya la haute société; sa différence de statut avec ses frères de couleur (toutes couleurs confondues) est sur ce point, évidente ${ }^{83}$. Mais s'il vécut « en égal parmi les grands » ${ }^{84}$, il rencontra aussi des obstacles : ce fut une manœuvre ouvertement basée sur la couleur de sa peau qui fit écarter sa nomination à la tête de l'Opéra de Paris (Académie royale de musique) ${ }^{85}$. Et au total sa réussite, même éclatante - en dépit d'une situation financière modeste qui l'amena, en Angleterre, à accepter de participer à des duels "arrangés" 86 - ne nous paraît pas contredire les résultats d'une étude portant sur les mulâtres nés à Basse-Terre, mais qui eux, étaient restés dans l'île : "les "sang-mêlé" gravissent peu à peu les échelons de la hiérarchie sociale. Mais une barrière infranchissable les sépare encore des Blancs à la veille de la Révolution ${ }^{87}$.

44 Tout cela, bien entendu, n'interdit pas aujourd'hui à la nation de le célèbrer. Encore faut-il que cela soit fait loyalement, et non avec un triomphalisme de mauvais aloi, comme sur cette plaque maintenant apposée dans le quartier de la Madeleine à Paris :

\section{« RUE DU CHEVALIER DE SAINT-GEORGE \\ (ANCIENNE RUE RICHEPANCE) \\ 1739-1799 \\ MUSICIEN ET CHEF D'ORCHESTRE \\ COLONEL DE LA GARDE NATIONALE »}

45 Passons, sur la date de naissance erronée. Passons encore sur ce «musicien et chef d'orchestre», dont la maladresse est d'autant plus flagrante que «musicien» (ou compositeur [ $\left.{ }^{88}\right]$ ) ici allait de soi : on aura voulu trop en faire, avec l'image publique qui s'attache à l'activité de chef d'orchestre... Mais c'est surtout du point de vue historien que l'on mène le promeneur par le bout du nez. «Colonel de la garde nationale »? Outre que c'est là une non-information (car il n'y a rien que de très normal à avoir fait partie de la garde nationale, pour qui participa à la Révolution française), on ne saurait mieux mentir par omission, plus de deux siècles après ce décret de décembre 1792 qui déjà avait ôté à la "Légion Noire » son identité ${ }^{89}$.

Enfin, il y a ce que recouvre cette plaque. En effet, l'objectif annoncé était de débaptiser la rue Richepance, ainsi dénommée d'après celui qui fut le "sabreur" de Bonaparte en Guadeloupe, et de la réaffecter à un héros de la lutte adverse, choisi parmi les victimes de la campagne de 1802. Plusieurs noms étaient en concurrence, dont celui de la mulâtresse Solitude. Mais celui de Saint-George est apparu comme étant plus apte à ne pas « réveiller les vieilles querelles ${ }^{90}$.

47 On est en droit de voir ici une directe conséquence de cette image de "nègre des Lumières » qui a pu être utilisée publicitairement, et qui, outre qu'elle ne manque pas de vulgarité, paraît appeler deux remarques :

48 - elle est par trop indulgente envers les Lumières qui, en dépit de leurs admirables énoncés (cf. le Chevalier de Jaucourt : "en un mot, rien au monde ne peut rendre l'esclavage légitime » $\left.{ }^{\left[{ }^{11}\right]}\right]$, eurent parfois quelque peine à briller de ce côté-là ; 
49 est anachronique, s'agissant d'un personnage qui n'entre dans la "grande Histoire » qu'après lesdites Lumières. Fouquier-Tinville, peu enclin d'ordinaire au compliment, n'hésita pas à affirmer qu'il eut, en une circonstance, l'occasion de « sauver ou perdre la république ${ }^{92}$. Ce chef de Brigade n'accomplissait sans doute rien que de très banal à ses propres yeux lorsque, témoin d'une péripétie qui trahissait un général proche de Dumouriez, il fonça au grand galop ${ }^{93}$ prévenir la municipalité de Lille; mais il n'en fallut pas plus pour éviter que la ville ne soit livrée à l'ennemi. Mais c'est au-delà de sa personne qu'il concerne l'historien de cette période : il incarne tous ces hommes et femmes, Noirs et mulâtres, qui, là où ils se trouvaient, participèrent à la Grande Révolution. Peu importe, qu'ils y aient participé à un moindre rang hiérarchique que le sien, l'important est qu'ils y aient participé à part entière.

\section{NOTES}

1.Journal de Paris, 26 prairial an VII, p. 1171. On consultera en priorité : Odet DENYS, Qui était le Chevalier de Saint-Georges?, Paris, Le Pavillon-Roger Maria, 1972 ; Emil F. SMIDAK, Joseph Boulogne nommé Chevalier de Saint-Georges, Lucerne, Fondation Avenira, 1996 (ouvrage de référence). Une notice biographique fiable figure dans l'éd. 2001 du New Grove Dictionary of Music and Musicians, v. 22, pp. 100-102.

2.Louise FusIL, Souvenirs d'une actrice, Dumont, Paris, 1841, t. 1, pp. 145-146.

3.Mercure français, n. 20, 20 germinal an V (6/4/1797), p. 105.

4.Mémoires du général Dumouriez, Ecrits par lui-même, Londres, s.e., 1794, t. 2, p. 99. À complèter par Edmond LeLEU, « La tentative de Dumouriez sur Lille en 1793 », Revue du Nord, t. IX, 1923, pp. 81-109.

5.Gabriel DEBIEN, Les Colons de Saint-Domingue et la Révolution, Paris, Armand Colin, 1953, p. 223.

6.Louise-Elisabeth VIGEE-LeBRUN, Souvenirs, Paris, Fournier, 1835, t. 1, p. 26.

7.Sur l'activité musicale de Saint-George à Lille durant cette période, d'utiles indications en B.M. Lille, Ms 1492 (papiers Léon Lefebvre).

8.Louis JАСОВ,» Saint-Georges et la "Légion Noire" de Lille en 1793 », Revue du Nord, t. XXXIII, 1951, pp. 6-17.

9.SHAT Xc 209, note préparatoire au décret du 6 décembre 1792.

10.Saint-George arriva à Hondainville à la mi-frimaire an II, soit au début de décembre 1793 ; cf. A.D. Oise, Archives communales Hondainville, 1D1. L'arrêté portant sa libération date du 2 brumaire an III (23/10/1794), mais il ne fut remis en liberté que le 27 prairial an III (15/6/1795) ; cf. A.N., F7 4551, "état" des pièces remises.

11.Pour registre des décès, cf. A.D. Seine, D.Q8 39.

12.Cf. notamment la belle notice de CuVELIER de TRIE dans le Courrier des spectacles du 24 prairial an VII, p. 2 (l'auteur, aujourd'hui oublié, fut un des créateurs du genre alors très populaire, qu'était le mélodrame).

13.Qui était le Chevalier de Saint-Georges?, op. cit., p. 32.

14.A.D. Nord, L 8532, 9 mai 1792.

Annales historiques de la Révolution française, 339 | janvier-mars 2005 
15.A.N., A 15 Col, 27 septembre 1776.

16.LA BoËSSIERE (fils), Traité de l'art des armes, Paris, Didot, 1818, p. XV ; Henry ANGELO, Angelo's Pic Nic; or Table Talk, London, Ebers, 1834 reprint 1905, p. 9. Les passages soulignés le sont par nos soins.

17. Biographie universelle ancienne et moderne, Paris, Michaud, 1825, t. XXXIX, p. 579.

18.Pierre LAROUSSE, Grand dictionnaire universel du XIX siècle, Paris, 1875, t. 14, p. 67.

19.Amédée de CAIX de SAINT-Aymour, Les Boullongne, Paris, Laurens, 1919, p. 92.

20.BACHAUMONT, Mémoires secrets pour servir à l'histoire de la République des Lettres en France, Adamson, Londres, 1780, t. XIV, pp. 43-44.

21.A.D. Gironde, 6B 51, 13 avril 1752.

22.SABATIER de CASTRES (attribué à), Les trois siècles de notre littérature, Amsterdam-Paris, Gueffier, Dehansi, 1772, t. I, p. 139.

23.Philippe de Roux, Le marquis de Casaux, Paris, Société de l'Histoire des Colonies Françaises, 1951, p. 11.

24.Cf. Mémoire Pour le Sieur Bologne de Saint-Georges, Écuyer, ancien Gentilhomme ordinaire du Roi. Contre le Sieur Vidal, Bourgeois de Paris, Paris, Cellot, 1764 (concerne un emprunt contracté imprudemment en 1762) ; A.N., A $15 \mathrm{Col}$ (concerne une action intentée contre la veuve et la fille de G. de Bologne, décédé en décembre 1774, pour paiement d'une grosse dette). On notera aussi que la fille n'accepta d'être reconnue héritière que "sous bénéfice d'inventaire", cf. A.N., X4b 736, 14 octobre 1775.

25.A.N., 01 101, 1er avril 1757.

26.BNF, Manuscrits, dossiers bleus 109, doss. 2666 Bologne.

27. À partir d'avril 1762, ce furent tous les Noirs et mulâtres, et non plus seulement les esclaves, qui furent tenus d'être déclarés; cf. Sue PEABODY, “There Are No Slaves in France", New York, Oxford University Press, 1996, p. 74. Dans le cas ici étudié la déclaration fut effectuée par La Boëssière, agissant au nom du sieur Bologne de SaintGeorges; cf A.N., Z1d 139, 12 mai 1762.

28.SHAT Yb 67, 15 juin 1764 ; l'indication “1761”, qui figure en SHAT Xk 9 (Formation du Régiment d'hussards américains \& du Midy, d'après le décrêt du sept septembre 1792), est erronée.

29. Mercure de France, février 1768, p. 13, et p. 215 (table); déjà signalé par DENYS, op. cit. 30.Une homonymie gênante, car elle concerne un autre suspect de 1793, a été signalée dans : Léon-Noël BERTHE, Dictionnaire des correspondants de l'Académie d'Arras au temps de Robespierre, Arras, Imp. Centrale de l'Artois, 1969, p. 189.

31.Alexandre DUMAS, notice biographique sur l'auteur, dans Roger de BEAUVOIR (pseud. de Edouard-Roger de Bully), Les soupeurs de mon temps, Paris, Faure, 1868, p. XVI.

32.Melesville (pseud. de Anne-Honoré-Joseph DuVEYRIER) et R. de BEAUVoIR, Le Chevalier de Saint-Georges, Paris, Mifliez et Tresse, 1840.

33.R. de BEAUVOIR, Le Chevalier de Saint-Georges, Paris, Delloye, 1840.

34.Cf. sur ce calcul qui ne fut pas toujours même conscient : Françoise VERGES, Abolir l'esclavage : une utopie coloniale, Paris, Albin Michel, 2001. L'idée que ce calcul était déjà présent à l'époque de la première abolition est au centre des travaux de Louis SALAMoLINS (cf. en particulier Les misères des Lumières, Paris, Laffont, 1992).

35.SAINT-GEORgES, Soldat Citoyen, de la ville de+++, Nous sommes donc trois?, ou le provincial à Paris, s.l., s.e., s.d. [mais 1790]. Le « provincial » du titre est l'auteur, maintenant lillois d'adoption. 
36.L'expression figure même dans un rapport d'indicateur ; cf. A.N., F/7/36883, 22 septembre 1793.

37. Courrier des spectacles, 24 prairial an VII, p. 2.

38.Henry GrÉGoIRE, De la littérature des Nègres, Paris, Maradan, 1808, p. 98.

39.Honoré de BALZAC, Le Cabinet des Antiques, Paris, Souverain, 1839, p. 81.

40.R. de BEAUVOIR, Le Chevalier de Saint-Georges, op. cit., pp. 5-6, note 1 (Avant-Propos). 41.Gabriel BANAT, « Le Chevalier de Saint-Georges, Man of Music and Gentleman-atArms : The Life and Times of an Eighteenth-Century Prodigy », Black Music Research Journal, v. 10 n. 2, fall 1990, p. 181 ; SMIDAK, op. cit., p. 102.

42.R. de BEAUVOIR, Le Chevalier de Saint-Georges, op. cit. (roman), p. 16.

43.Le Mémoire Pour le Sieur Bologne de Saint-Georges, op. cit., mentionne à la date de 1764 trois habitations (p. 2), soit « deux sucreries et une cafféyere » (p. 5).

44.Oruno D. LARA, « Le procès de résistance des nègres de Guadeloupe : guérilla et conspiration des nègres cimarrons, 1736-1738 ", Cimarrons, n. 1, 1981, pp. 13-78 ; Lucien-René ABENON, « La révolte avortée de 1736 et la répression du marronnage à la Guadeloupe », Bulletin de la société d'histoire de la Guadeloupe, n. 55, 1er trim. 1983, pp. 51-73.

45.ANGELo, op. cit., p. 9. Le mot Africa est ici utilisé pour désigner une origine - Nanon était née en Guadeloupe.

46.Lionel de LA LAURENCIE, L'École française de violon, v. II, Paris, Delagrave, 1923, pp. 449-500. Une ébauche parut en anglais : "The Chevalier de Saint-George », 1919, The Musical Quarterly, n. 1, pp. 74-85.

47.LA LAURENCIE, op. cit., v. II, p. 460. Cette phrase est moins accablante pour l'auteur, dont tout est là pour attester le dégoût que lui inspirait le racisme, qu'elle ne l'est pour une certaine France, dont il tentait ici de devancer les préjugés - et avec l'effroyable maladresse, que chacun est à même de constater.

48.Pour dossier personnel, cf. SHAT, 2Ye 1791/1847, doss. Saint-Georges Joseph. 49.LA LAURENCIE, op. cit., v. II, p. 449.

50.Id., p. 489.

51.A.D. Gironde, 9J 322, Fonds d'Arlot de Saint-Saud, dossiers de Boisserie de Masmontet ; doss. « de Boulogne », 23 mars 1935. Le passage souligné, l'est sur l'original.

52.Gaston BOURGEOIS, « Le Chevalier de Saint-Georges : inexactitudes commises par ses biographes ", Bulletin de la société d'histoire de la Guadeloupe, n. 2, 1964, pp. 12-17. Le même article annonce la parution prochaine de la biographie, par l'auteur, mais ce projet resta sans suite.

53.LA LAURENCIE, op. cit., v. II, p. 452 (l'auteur indique avoir eu accès, au manuscrit de Vauchelet).

54.Qui était le Chevalier de Saint-Georges?, op. cit.

55.Dix-Huitième siècle, n. 6, 1974, p. 378 (note de lecture) ; idem, n. 7, 1975, p. 434

(réponse de O. Denys).

56.DENYS, op. cit., p. 30. Pour rappel : ces additifs, du type Saint-Georges, n'étaient pas liés à une origine noble ; cf. VAUCHELET, Dugommier, Montreuil-sur-Mer, Imp. de "La Montreuilloise", 1899, p. 8.

57.DENYS, op. cit., p. 205 (et acte de décès en A.N., 5 Mi 132 SOM, 26 décembre 1774). 58.Cf. note 1, supra. L'édition anglaise est parue simultanément, sous le titre Joseph Boulogne called Chevalier de Saint-Georges. 
59.SMIDAK, op. cit., p. 99.

60.Alain GUEDE, Monsieur de Saint-George, Arles, Actes Sud, 1999, p. 64.

61.Sur ce parcours, cf. Jean-Baptiste du TERTRE, Histoire générale des Antilles habitées par les Français, Paris, Jolly, 1667, t. I, pp. 460-465 ; Gérard LAFLEUR, « L'origine des protestants de Guadeloupe au XVII ${ }^{\mathrm{e}}$ siècle », Bulletin de la société d'histoire de la Guadeloupe, n. 37, 1978, pp. 49-57.

62.G. LAFLEUR, « Les protestants de la société antillaise des origines à nos jours ", Études Guadeloupéennes, n. 5, 1992, p. 111.

63.A.N., C8a $18 \mathrm{Col}, 26$ juin 1711 (déjà cité par G. LAFLEUR dans le Bulletin de la société d'histoire de la Guadeloupe, $n^{\circ}$ spécial, « Les Protestants aux Antilles françaises du Vent sous l'Ancien Régime », 1987, p. 294).

64.A.N., 5 Mi 138 SOM, 26 janvier 1740.

65.G. LAFLEUR, « Le pouvoir et les protestants de la Guadeloupe aux XVII ${ }^{\mathrm{e}}$ et XVIII siècles », Bulletin de la société d'histoire de la Guadeloupe, n. 39, 1979, p. 39.

66.L'inquiétude était ici légitime : le jugement rendu le 31 mai 1748 condamnait G. de Bologne, outre à des peines inexécutables en son absence (dont la mort par pendaison), à la confiscation immédiate de ses biens ; cf. CAOM (Aix-en-Provence), doss. E 37 famille de Bologne Guadeloupe.

67.A.N., B 89 Col.

68.Ibid., F5b $39 \mathrm{Col}$.

69.Ibid., F5b $14 \mathrm{Col}$.

70.A.D. Gironde, Fonds de l'Amirauté de Guïenne 6B 396.

71.Ibid., 6B 50, 1er août 1749. Les concubines, et même les « favorites ", étaient loin d'avoir toujours droit à tant d'égard. On pourra lire Arlette GAUTIER, Les sœurs de Solitude, Paris, Éd. Caribéennes, 1985.

72.GRÉGOIRE, De la noblesse de la peau, ou du préjugé des Blancs contre la couleur des Africains et celle de leurs descendans Noirs et Sang-mêlés, Paris, Baudouin, 1826, p. 21.

73.Florence GAUTHIER, « Y a-t-il une politique des colonies en l'an II ? ", Annales historiques de la Révolution française, n. 300, avr.-juin 1995, pp. 227-28.

74.La demande des volontaires de couleur fut présentée en séance à l'Assemblée, et aussitôt acceptée, le 7 septembre 1792 ; cf. Le Moniteur universel du 9 septembre, p. 1074. 75. Claude PERROUd, « Notice sur la vie de Brissot », dans Jacques-Pierre BRISSOT, Correspondance et Papiers, publiés par C. Perroud, Paris, Picard, s.d. [1911], p. XIV. 76.Le deuxième séjour de Saint-George à Londres est celui de 1789-1790. Sa présence dans cette capitale est attestée dès le mois de mars de l'année 1789 (cf.

«Correspondance de Philidor. Londres, 1783-1795 », dans Recherches sur la musique française classique, t. XXVIII, 1993-1995, p. 134, lettre du 17 mars 1789). Nous n'aurons pas de mots assez durs pour un auteur qui ne doit pas espèrer ici d'autre publicité, et qui a récemment insinué qu'il aurait quitté la France... après la prise de la Bastille. 77.DEBIEN, op. cit., p. 223. La vocation de Saint-George à effectuer des missions sensibles peut expliquer son absence, par souci de discrétion, de correspondances où on pourrait s'attendre à le voir figurer.

78.A.C. Lille, 17882 doss. 25. Pour rappel : le mot « patriote », en cette année 1793, est à considérer au sens le plus large - défense de la Révolution.

79.Dans sa déclaration (note 27, supra) La Boëssière indique que son élève avait débarqué « un an avant la guerre », ce qui fait bien 1755 : la guerre en question est celle de Sept Ans, qui débute en 1756. 
80.A.N., F5b 14 Col, 26 août 1755.

81.Ibid., Z1d 139, 10 mai 1762.

82.A.D. Nord, L 8072, 15 mai 1793.

83.La différence de statut entre Saint-George et la plupart des Noirs et mulâtres alors présents en métropole est au centre d'une fiction du romancier Roland Brival : Le Chevalier de Saint-Georges, J-C Lattès, 1991.

84.DenYs, dans Hommes et Destins, Académie des Sciences d'Outre-Mer, Paris, 1981, t. IV, p. 630 (notice ad nomem, qui nous a été aimablement signalée par Mr Patrick Martin). 85.GRIMM et DIDEROT, Correspondance littéraire, philosophique et critique, Paris, Buisson, 1813, 3e partie, t. 1 , pp. 84-85.

86. Michael KeLLY, Reminiscences, London, Colburn, 1926, t. I, p. 344. Obtenir même un résultat nul face à Saint-George, était un "événément" ; cf.» Correspondance de Philidor. Londres, 1783-1795 », art. cit., p. 170, lettre du 8 juin 1790.

87.Frédéric RÉGENT, «Couleur, statut juridique et niveau social à Basse-Terre (Guadeloupe) à la fin de l'Ancien Régime (1789-1792) », dans Jean-Luc BonNiol (dir.), Paradoxes du métissage, Paris, Éd. du C.T.H.S., 2001, p. 50. On consultera, sur le préjugé lié à la couleur, dans le contexte antillais : BonNiol, La couleur comme maléfice, Paris, Albin Michel, 1992.

88.Pour la musicographie de Saint-George, cf. Dominique-René de LERMA, « Black Composers in Europe : A Works List », Black Music Research Journal, v. 10 n. 2, fall 1990, pp. 319-332.

89. Concernant la garde nationale, nous avons nous-même rappelé au début du présent article que ce citoyen y servit (comme capitaine). Pour le reste, ce fut bien entendu à l'armée qu'il devint colonel, et ce pour commander la légion ici volatilisée.

90.L'objection au nom de laquelle Saint-George n'était déjà plus de ce monde en 1802 fut écartée par les édiles, soulagés par cette solution venue préserver la physionomie traditionnelle du quartier : on décrèta que l'intéressé aurait pu - on a bien lu -, faire partie des victimes de Richepance (pour « exposé des motifs ", cf. site informatique www.paris.fr ; Conseil Municipal, Débats, séance des 17 et 18 décembre 2001).

91.DideROT et D'ALEMBERT, Encyclopédie ou Dictionnaire raisonné des Sciences, des Arts et des Métiers, Paris, Briasson-David-Le Breton-Durand, 1755, t. V, p. 938 (notice Esclavage). 92.Bulletin du Tribunal criminel révolutionnaire, n. 38, p. 151 (séance du 17 mai 1793); l'accusateur public s'adressait ici à Saint-George lui-même, comparaissant comme témoin, cf. A.N., W 271 doss. 31 Miaczinski. Nous sommes en désacord avec une affirmation souvent reprise depuis LA LAURENCIE (op. cit., v. II, p. 477) et selon laquelle le contexte de dénonciation l'aurait amené, le même mois, à se justifier devant ce même Tribunal.

93.Les témoignages attestent la célérité dont fit preuve Saint-George, ce matin du 2 avril 1793 : la carence qui lui est attribuée par le romancier Claude RIBBE (Alexandre Dumas, le dragon de la reine, Monaco, Éd. du Rocher, 2002, pp. 131-34) est sans fondement. 


\section{RÉSUMÉS}

Le caractère lacunaire des archives anciennes de la commune du Baillif (Guadeloupe) et la naissance illégitime de Saint-George, enfant naturel d'une esclave, expliquent en partie le flou qui a pu entourer l'état-civil de celui-ci : trois dates de naissance différentes ont pu lui être attribuées, non sans quelque bien-fondé pour chacune. Mais, en ce qui concerne les paternités qui ont été successivement mises en avant - et sans jamais de preuve à l'appui : il s'agissait au départ, pour une société incapable de concevoir qu'un mulâtre aît pu avoir du talent, d'en faire malgré lui le fils d'un aristocrate. Ce type de motivation n'a plus cours, en principe, et le vrai père est connu depuis 1972 ; mais on assiste encore à des tentatives pour brouiller les cartes.

A certificate of birth, full of stake: Saint-George, 1745-1799. The ancient archives of the Baillif, civil parish in Guadeloupe, being incomplete and the illegitimate birth of Saint-George, who was a slave's natural child, account for the vagueness Saint-George's certificate of birth. Indeed he was given three different dates of birth, all being fully-justified. However, concerning his affiliation, living in a society incapable of imagining that a mulatto could be gifted, he was made to be son of an aristocrat. Actually his real father was known in 1972. But there are still attempts to spread confusion.

INDEX

Mots-clés : Saint-George, hommes de couleur, discrimination, filiation, état-civil

\section{AUTEUR}

LUC NEMETH

Docteur en histoire contemporaine

BP 6343

75063 Paris Cedex 02 